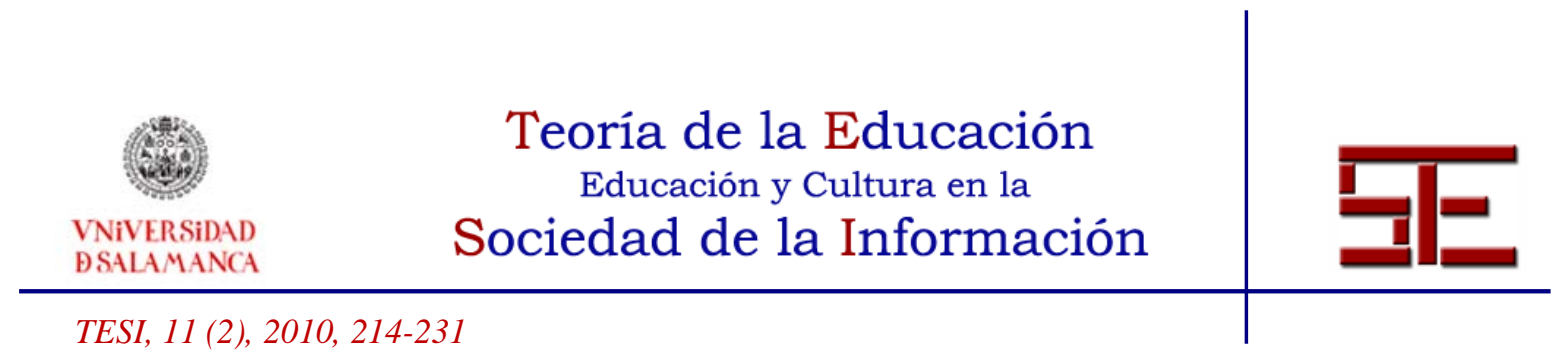

\title{
RECURSOS TECNOLOGICOS AUDIOVISUALES DE FORMACIÓN EN RED: SISTEMAS STREAMING MEDIA Y TELEINMERSIVOS
}

Resumen:

Presentamos dos modalidades tecnológicas de enseñanza en red: la consolidada y ampliamente utilizada tecnología video-streaming, y el futuro de la comunicación a distancia, la teleinmersión.

La primera, permite la transmisión de audio/video por la red para que puede ser vista por el usuario en su ordenador personal, desde cualquier lugar que disponga de una conexión a red. La información será recibida y decodificada por el usuario final utilizando cualquier reproductor que existen en el mercado.

La teleinmersión, por su parte, permite crear espacios virtuales de colaboración entre profesionales, ofreciendo entornos muy cercanos a la realidad. Esta tecnología revolucionará, sin duda, nuestros sistemas de enseñanza en los próximos años, facilitando la interacción profesor-alumno.

Es evidente que la formación e-learning, aporta a los alumnos y a los docentes grandes ventajas como: menores tiempos de aprendizaje, flexibilidad de horarios y de ubicación geográfica, entre otras.

Palabras clave: Formación a distancia, video streaming. Teleinmersión. Tecnologías de la información

Juan Antonio Juanes Méndez, $M^{a}$ Auxiliadora Velasco Marcos, Francisco Javier Cabrero Fraile, José Miguel Sánchez Llorente v $M^{a}$ José Rodríauez Conde. 


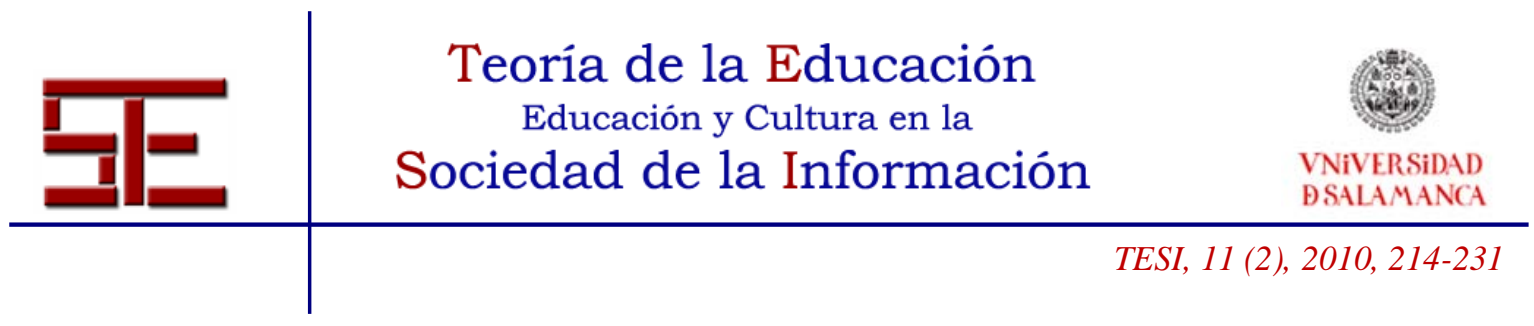

\title{
TECHNOLOGICAL RESOURCES FOR ON-LINE INSTRUCTION: STREAMING MEDIA AND TELEIMMERSION SYSTEMS
}

\begin{abstract}
:
We describe two technological modes of on-line teaching: the consolidated and widely used video-streaming mode and teleimmersion, the future of distance communications. The former mode allows the transmission of audio/video through the network so that it can be seen by the user on a PC from anywhere harbouring a network connection. The information is received and decoded by the final user using any reproducer available on the market.

Teleimmersion allows the creation of virtual spaces for collaboration among professionals, offering venues that are very similar to reality. This technology will undoubtedly revolutionize our teaching systems in the near future, facilitating instructor-student interaction.

It is clear that e-learning- instruction offers both students and instructors huge advantages, such as shorter learning times and schedule and geographic flexibility, among others
\end{abstract}

Key words: Distance learning, video streaming. Teleimmersion. Information Technologies.

Juan Antonio Juanes Méndez, $M^{a}$ Auxiliadora Velasco Marcos, Francisco Javier

Cabrero Fraile, José Miguel Sánchez Llorente y $M^{a}$ José Rodríguez Conde

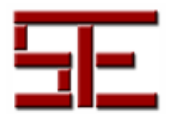




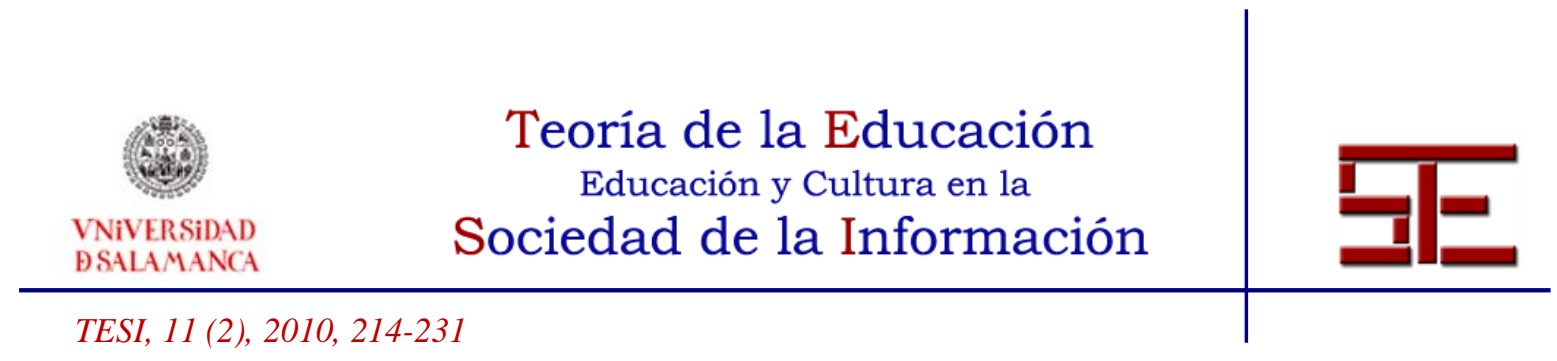

\title{
RECURSOS TECNOLOGICOS AUDIOVISUALES DE FORMACIÓN EN RED: SISTEMAS STREAMING MEDIA Y TELEINMERSIVOS
}

Fecha de recepción: 18/09/2009; fecha de aceptación: 15/05/2010; fecha de publicación: 05/07/2010

\author{
Juan Antonio Juanes Méndez \\ jajm@usal.es \\ Universidad de Salamanca \\ Ma Auxiliadora Velasco Marcos \\ mauxi.velasco@consalamanca.es \\ Centro de salud de Tejares. Salamanca \\ Francisco Javier Cabrero Fraile \\ cabrero@usal.es \\ Universidad de Salamanca \\ José Miguel Sánchez Llorente \\ jmsll@usal.es \\ Universidad de Salamanca \\ $\mathrm{M}^{\mathrm{a}}$ José Rodríguez Conde \\ mjrconde@usal.es \\ Universidad de Salamanca
}

\section{1.- INTRODUCCIÓN}

La combinación eficaz de sistemas avanzados de telecomunicaciones con la utilización de tecnologías multimedia de última generación, están permitiendo transmitir información desde puntos distintos distribuidos geográficamente.

Para la formación académica de nuestros alumnos, intentamos aprovechar todas las ventajas potenciales del uso de las Tecnologías de la Información y la Comunicación (TIC) en la docencia, no sólo universitaria, sino también en la formación de médicos internos residentes hospitalarios, y los distintos profesionales, mediante cursos de formación continua.

El profesor, como transmisor de conocimientos, debe tener una actualización permanente no solo de los contenidos docentes a transmitir, sino estar preparado para

Juan Antonio Juanes Méndez, $M^{a}$ Auxiliadora Velasco

Marcos, Francisco Javier Cabrero Fraile, José Miguel

Sánchez Llorente v $M^{a}$ José Rodríauez Conde.

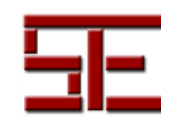




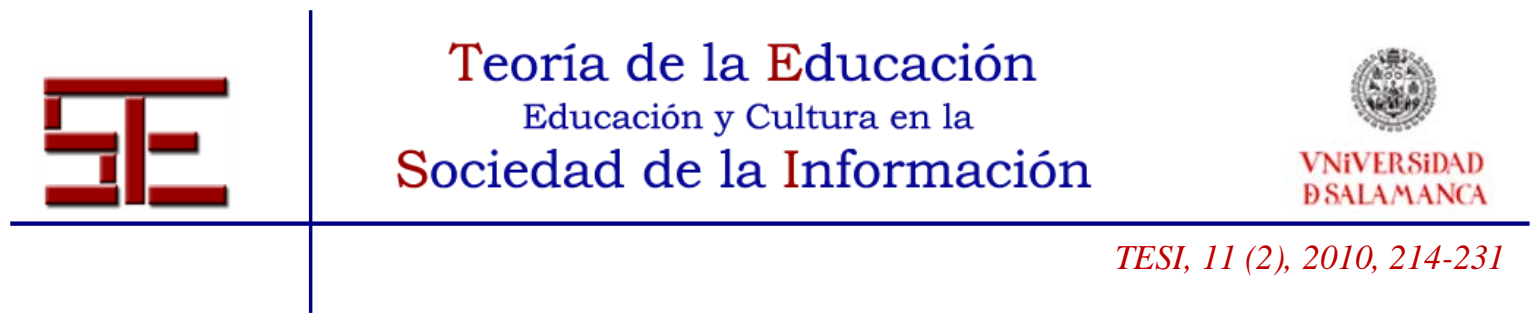

diseñar nuevas estrategias de aprendizaje y dirigiendo al universitario en su labor formativa.

Las TIC pueden contribuir a la mejora del proceso de formación en el campo de las ciencias de la salud, como en otras áreas de conocimiento, lo que conlleva a conocer bien los diferentes medios tecnológicos que se utilizan para la comunicación entre profesores-alumnos, tutores-residentes o médicos-pacientes; ello permitirá extraer pautas de trabajo positivas entre ambos, suponiendo una mejora y un aumento de la calidad en la formación.

El acelerado avance tecnológico en nuestra sociedad, está provocando grandes transformaciones en los sistemas de comunicación, que posibilitan llevar a cabo metodologías adicionales de formación y atención a distancia, en tiempo real, gracias al empleo de tecnologías de interrelación personal.

Cada día están surgiendo nuevas plataformas informáticas como estructuras coordinadas de software y hardware que proveen la base para la construcción de sistemas de información para la enseñanza y la formación, en multitud de disciplinas médicas.

\subsection{Tecnología video streaming}

Una de las tecnologías que juega un papel importante, en la formación a distancia, es la tecnología bajo entorno video-streaming, la cual consiste en la transmisión tanto de audio como de video por redes IP (como es Internet), para que pueda ser visto por el usuario en su ordenador personal. El contenido tanto de audio como de video, ya sea en vivo o grabado con anterioridad, se encuentra codificado, es decir, digitalizado y comprimido; y es transmitido por Internet o Intranet como paquetes de información. La información será recibida y decodificada por el usuario final usando un reproductor (Windows Media Player, Quick Time, Real Player).

El termino streaming significa corriente o flujo, lo que determina que sea una tecnología que permite la recepción instantánea, sin esperas, de información que fluye desde un servidor.

Esta tecnología surge de la necesidad de acceder a tipos de información voluminosa que generan amplios tiempos de espera usando la tradicional descarga de archivos. Esta información es, fundamentalmente, de tipo audiovisual aunque puede ser sólo audio o vídeo. Es conveniente diferenciar entre lo que es video streaming y la transmisión de video cotidiana por Internet. Los métodos tradicionales para la entrega de video IP

Juan Antonio Juanes Méndez, $M^{a}$ Auxiliadora Velasco Marcos, Francisco Javier Cabrero Fraile, José Miguel Sánchez Llorente y $M^{a}$ José Rodríguez Conde
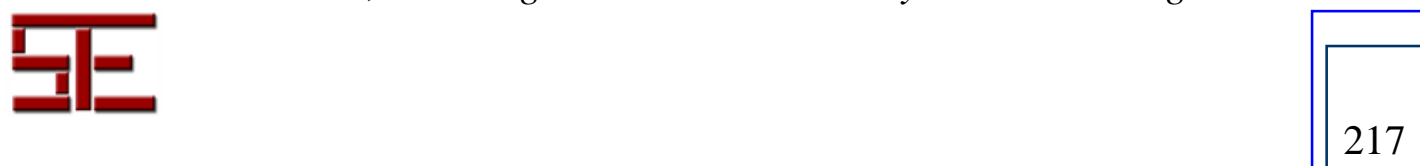


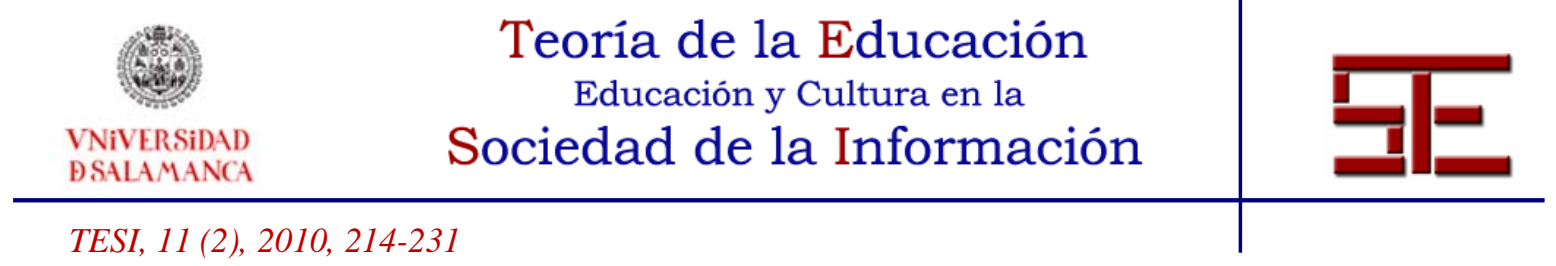

requieren descargar el video completo a su computadora personal antes de poder verlo. En la mayoría de los casos este proceso es muy lento y ocupan una gran cantidad del disco duro y del ancho de banda disponible. El video streaming, por el contrario, no requiere información para descargar y la reproducción del contenido es casi inmediata.

En ocasiones surgen preguntas sobre las diferencias entre lo que es la transmisión de video por Internet, el video bajo demanda, la video conferencia y el video streaming.

La transmisión de video utiliza redes de IP que envían información por medio de stream a un número ilimitado de usuarios que lo estén viendo simultáneamente. El video bajo demanda ofrece la posibilidad de acceder a los programas que han sido pregrabados y archivados. Un segmento de información de streaming por programa es necesario para cada usuario. La video conferencia es un escenario que permite a los usuarios colaborar en tiempo real con otros que se encuentren en otros lugares que tengan hardware dedicado que corre sobre un ancho de banda de red intensiva.

El video streaming es una eficiente forma de transmisión que puede alcanzar un ilimitado numero de usuarios y altamente escalable, creando un escenario "cualquier hora y cualquier lugar" y pudiendo soportar los tres tipos de distribución de video.

Por otra parte, mientras el video es transmitido, otros elementos pueden ser presentados en recuadros, pudiendo incluir texto, gráficas y animaciones sincronizadas con la transmisión de video. Se puede adaptar con presentaciones de Power Point o archivos de red portátiles como los HTML, JPEG y GIF.

Todos estos elementos están reunidos en la ventana del browser mientras se realiza la transmisión.

La tecnología de streaming se utiliza para aligerar la descarga y ejecución de audio y vídeo en la web, ya que permite escuchar y visualizar los archivos mientras se están descargando. Si no utilizamos streaming, para mostrar un contenido multimedia en la Red, tenemos que descargar primero el archivo entero en nuestro ordenador y más tarde ejecutarlo, para finalmente ver y oir lo que el archivo contenía. Sin embargo, el streaming permite que esta tarea se realice de una manera más rápida y que podamos ver y escuchar su contenido durante la descarga.

Actualmente y gracias a las comunicaciones de banda ancha disponibles (ya sea DSL, Cable, Satélite) y a los sistemas de compresión de audio y vídeo con calidad (mp3, mpeg-4, divx, etc.) se ha hecho mucho más fácil descargarse grandes cantidades de información audiovisual. Por eso, se pensó en una manera, tecnológicamente posible, de retransmitir con facilidad esa información y el resultado fue la tecnología del streaming.

Juan Antonio Juanes Méndez, $M^{a}$ Auxiliadora Velasco Marcos, Francisco Javier Cabrero Fraile, José Miguel Sánchez Llorente v $M^{a}$ José Rodríauez Conde.

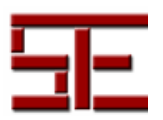




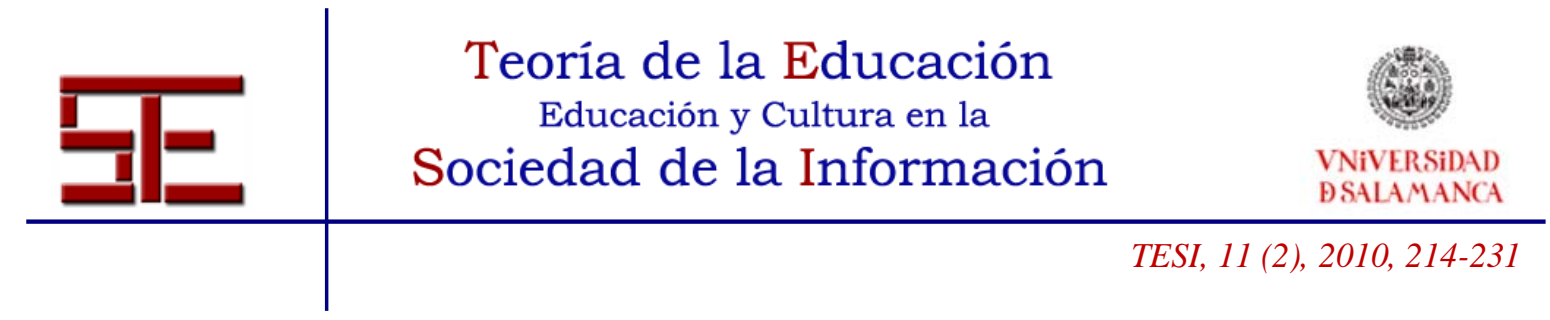

Como se ilustra en el esquema, la instalación de un servicio de "video streaming" convencional contiene dos actividades fundamentales y bien diferenciadas: la elaboración de contenidos en un formato digital, utilizando procedimientos de compresión, y la distribución de los contenidos por la red a los clientes o usuarios finales.

Para la elaboración de contenidos es necesaria en primer lugar la captura de audiovideo; y una segunda fase, se llevará a cabo el proceso de compresión en la que se trata separadamente el audio y el video. El resultado de esta actividad puede ser un fichero multimedia, o una corriente (streaming).

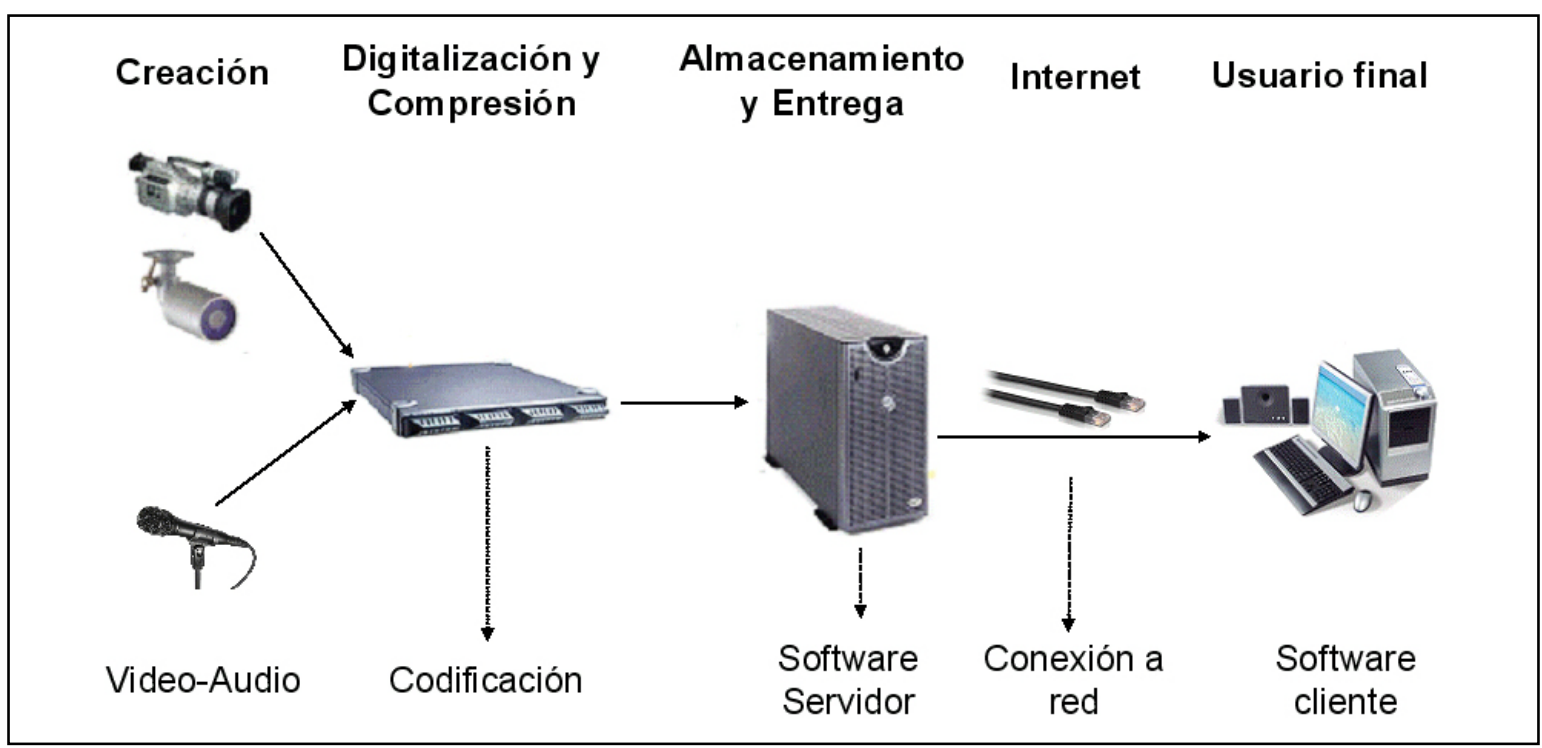

Esquema básico que representa e ilustra los pasos más relevantes a seguir para el desarrollo e implantación de la tecnología video-streaming, desde su elaboración hasta su distribución por red para llegar al usuario final.

La distribución de contenidos incluye la difusión de las referencias, generalmente mediante URLs de los mismos incluidos en páginas web. Un servidor especializado (servidor de streaming), almacena y/o distribuye los contenidos a los clientes. Los servidores pueden proporcionar dos tipos de contenidos:

Juan Antonio Juanes Méndez, $M^{a}$ Auxiliadora Velasco Marcos, Francisco Javier Cabrero Fraile, José Miguel Sánchez Llorente y $M^{a}$ José Rodríguez Conde

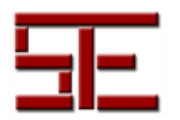




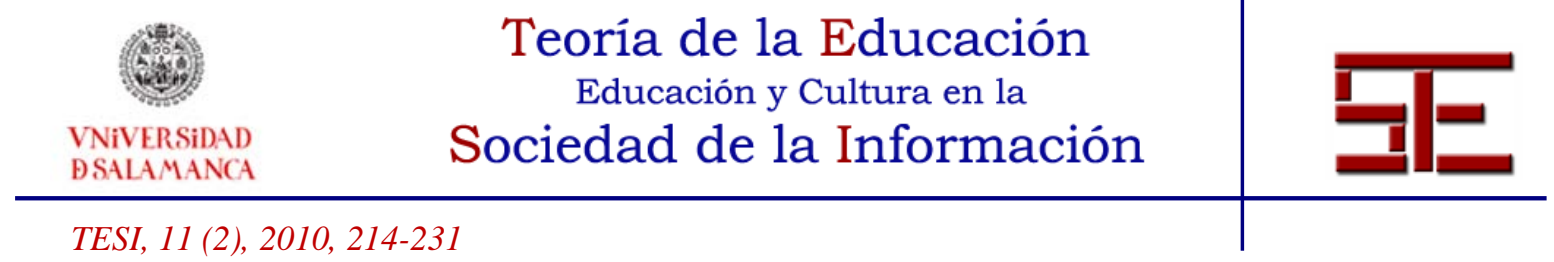

- VoD (video on demand), petición por clientes individuales de ficheros almacenados en el servidor, sobre los que tiene un control similar a un video doméstico (posicionamiento, paro, retroceso o avance rápido...).

- Difusión (broadcast) a varios clientes de un mismo contenido, ya sea creado en ese momento en vivo (live broadcast), o almacenado previamente en el servidor.

Para ofrecer contenidos multimedia siguiendo el modelo VoD (video on demand) es posible utilizar servidores web convencionales. Como principal ventaja se reduce la complejidad de la instalación, ya que un servidor WWW es un elemento conocido. Sin embargo, no se considera una elección adecuada, porque los ficheros multimedia son de gran tamaño, y consumen tiempo de CPU y de comunicaciones por largos períodos de tiempo. A esto se podría sumar además, la posible acumulación de peticiones simultáneas de un mismo contenido.

La opción más adecuada es instalar un servidor especializado ya que son capaces de ofrecer servicios de difusión (broadcast) que no están disponibles en los servidores web convencionales, utilizando cuando es posible tecnologías IP multicast con lo que esto significa de ahorro de ancho de banda.

Para poder recibir y ejecutar los archivos de streaming a través de Internet, se requiere disponer tan solo de un software de cliente.

RealPlayer es el formato más utilizado en el mundo del streaming. Es una aplicación ampliamente extendida y es uno de los plug-in más normales de cualquier explorador. Está disponible para múltiples plataformas y se puede encontrar en paquetes que incluyen todas las herramientas necesarias para poner en funcionamiento un sistema de streaming.

Otro formato empleado es QuickTime Player, siendo su principal ventaja el ser un servidor de streaming gratuito, estando disponible para entornos Windows y Unix.

Finalmente, señalamos el formato de Windows Media, creado por Microsoft, y e limitado al entorno Windows, por lo que utiliza formatos propios. Su principal ventaja es que todas las herramientas son gratuitas.

La tecnología video streaming puede ser desarrollada sobre cualquier infraestructura de red actual y sólo necesita un mínimo de elementos instalados en un ordenador. Los requisitos mínimos necesarios para habilitar video-streaming son los que actualmente cualquier casa comercial ofrece en sus equipos informáticos:

Juan Antonio Juanes Méndez, $M^{a}$ Auxiliadora Velasco Marcos, Francisco Javier Cabrero Fraile, José Miguel Sánchez Llorente v $M^{a}$ José Rodríauez Conde. 


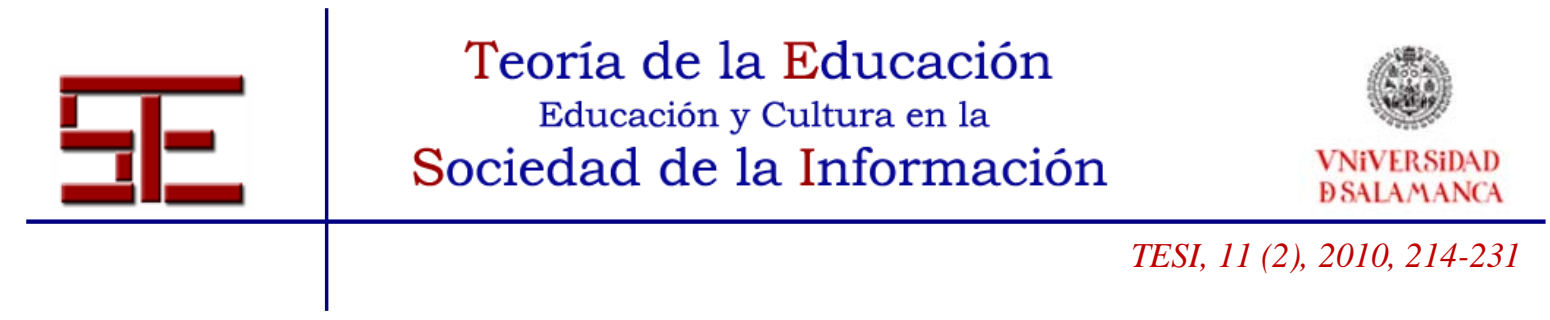

En la mayoría de los casos, el número de personas que pueden acceder a esta tecnología al mismo tiempo, depende del ancho de banda, tráfico de red, poder del servidor de streaming y número de licencias de streaming disponibles. En caso de un evento en vivo, estos factores determinarán cuántos usuarios finales pueden acceder. Además, los eventos pueden ser archivados para transmisión bajo demanda.

El video-streaming está teniendo una infinidad de aplicaciones, las cuales aumentan a medida que la tecnología progresa. La tecnología video-streaming se usa predominantemente en:

* Comunicados de empresas a gran escala incluyendo juntas virtuales, entrega de noticias y entrenamientos.

* Entretenimiento (transmisiones de deportes, música y cine).

* Publicidad.

* Educación y aprendizaje a distancia. Formación continuada.

\section{2.- EXPERIENCIA DIDÁCTICA EN EL CAMPO DE LAS CIENCIAS DE LA SALUD, BAJO EN ENTORNO VIDEO STREAMING}

Los cambios en el proceso de enseñanza-aprendizaje, en el marco del Espacio Europeo de Educación Superior (EEES), nos ha obligado a revisar la metodología de enseñanza y el diseño curricular, buscando estrategias que motiven al aprendizaje autónomo, empleando para ello herramientas didácticas dirigidas y coordinadas por el profesor. En este sentido, hemos llevado a cabo una experiencia en red empleando la tecnología video streaming, para el estudio de la anatomía radiológica del Sistema Nervioso Central a través de diferentes técnicas de diagnóstico por imagen. El curso desarrollado permitió su acceso, desde cualquier parte del mundo con conexión a Internet. Cada sesión tuvo una duración estimada no superior a los 45 minutos, pudiendo repetirla el usuario cuantas veces considerara oportunas, y a la hora que le fuera conveniente.

En todo momento los usuarios pudieron mantener contacto con los profesores que impartían los diferentes temas, a través del correo electrónico, para cualquier aclaración, duda o problema, que pudiera surgir a lo largo del período de duración del curso.

Juan Antonio Juanes Méndez, $M^{a}$ Auxiliadora Velasco Marcos, Francisco Javier

Cabrero Fraile, José Miguel Sánchez Llorente y $M^{a}$ José Rodríguez Conde
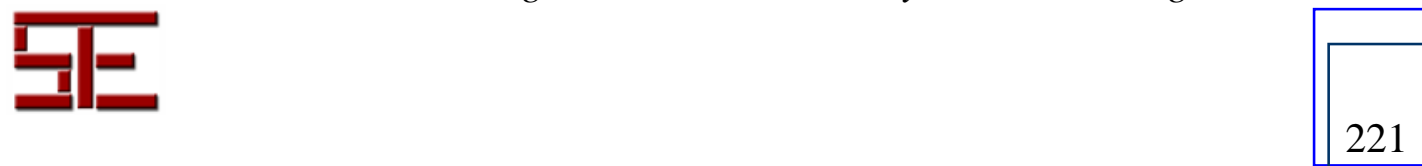


Teoría de la Educación
$\begin{gathered}\text { Educación y Cultura en la } \\ \text { VNiVERSADAD } \\ \text { BSALAMANCA }\end{gathered}$

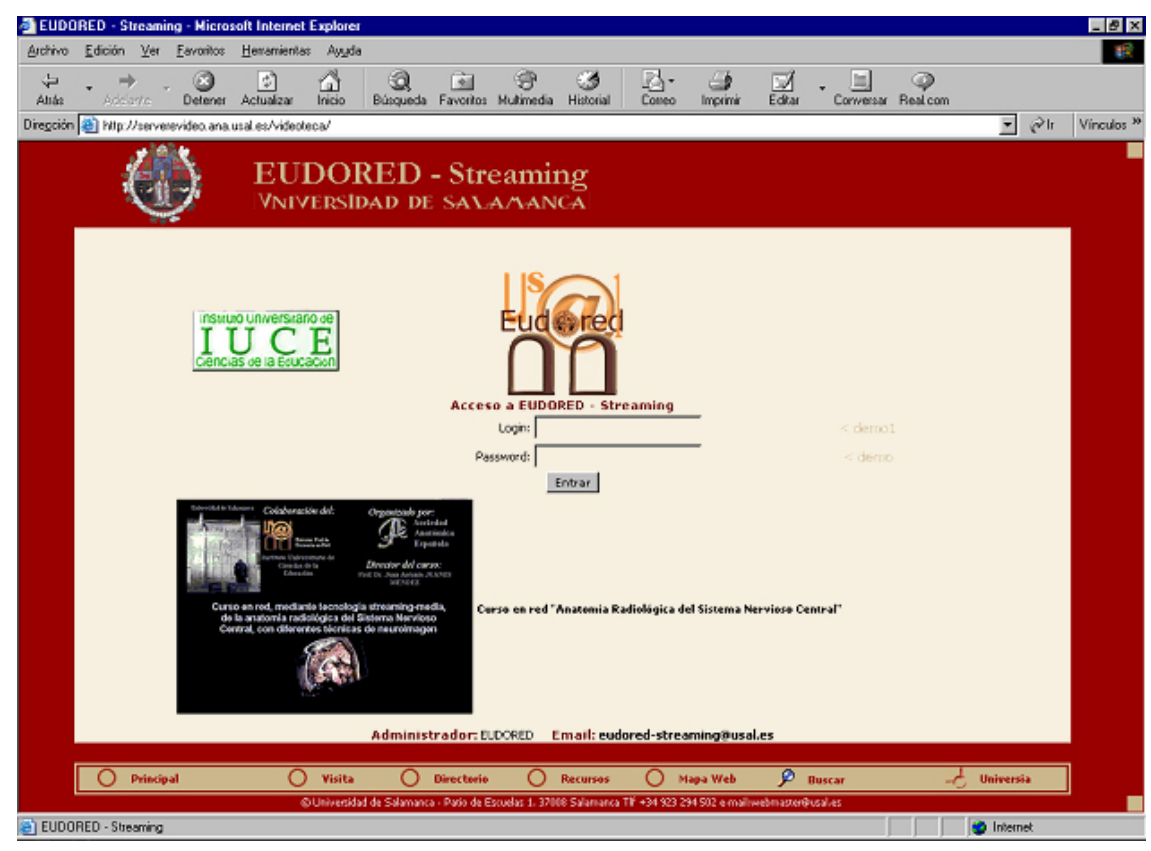

Página, de entorno de educación en red, del Instituto Universitario de Ciencias de la Educación, de la Universidad de Salamanca. Pantalla de acceso al curso, mediante el Login y Password asignados a los usuarios.

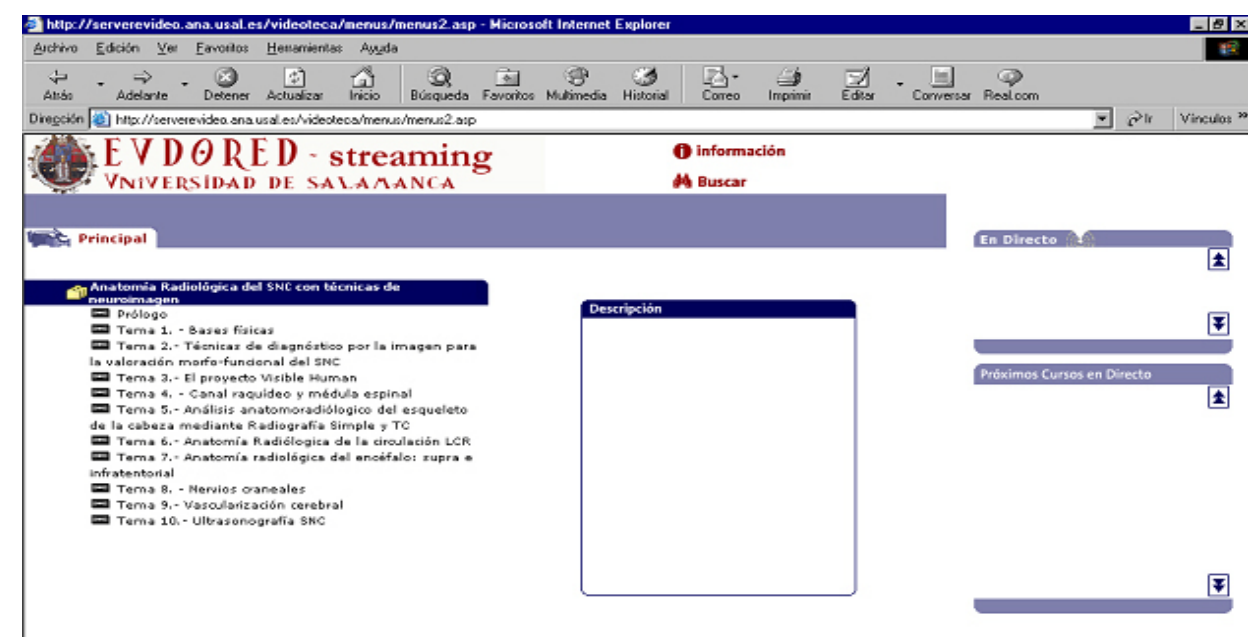

Despliegue de los diferentes temas que configuran el curso en red, a los que el usuario puede acceder libremente, en el orden que desee. 


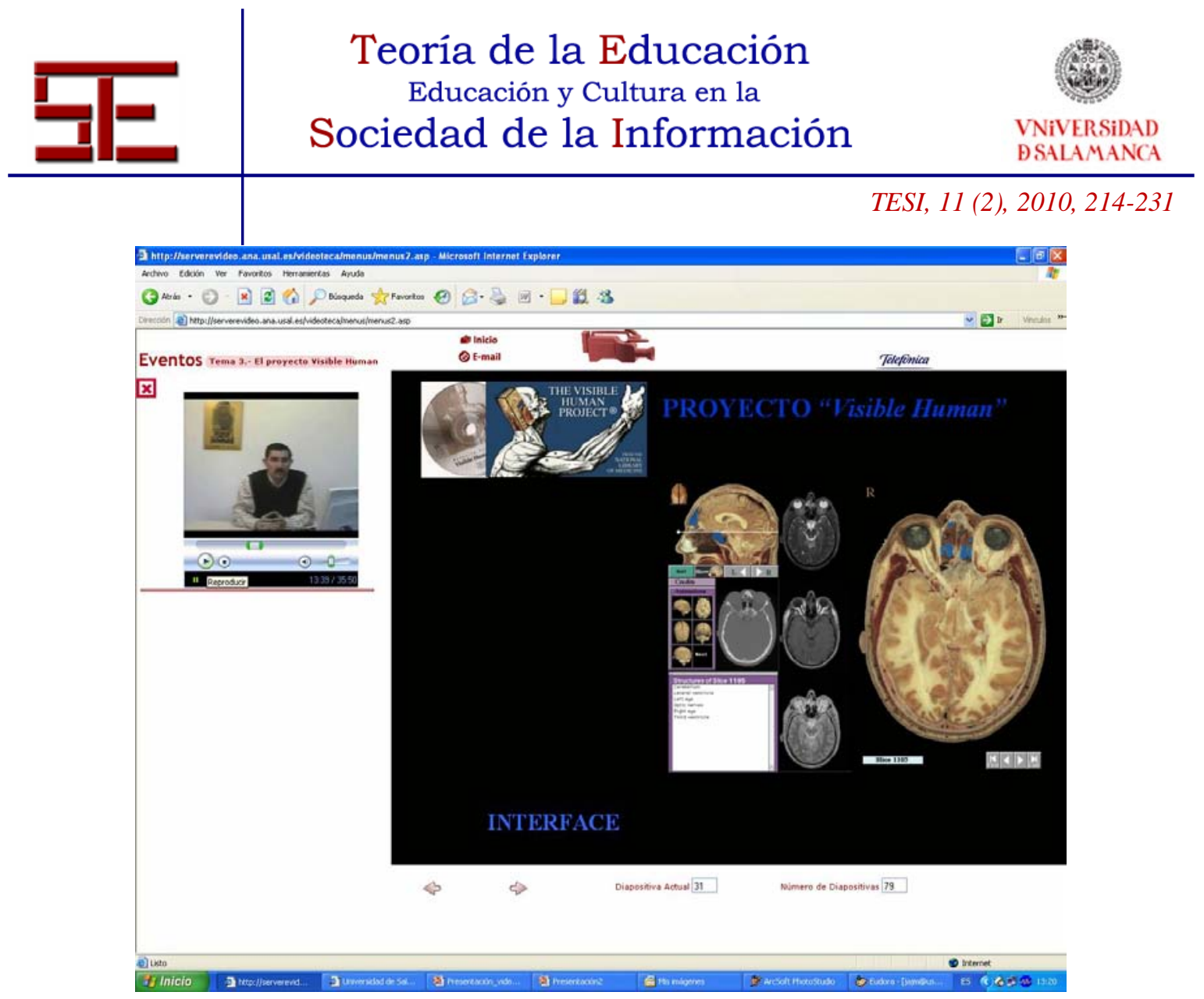

Tras la exposición y visualización de todos los temas del curso se llevó a cabo un una evaluación, mediante un cuestionario de preguntas tipo test, así como la descripción de alguna imagen radiológica, para valorar los conocimientos adquiridos durante el desarrollo del curso. Los resultados conseguidos fueron muy satisfactorios en la mayor parte de los usuarios inscritos al curso.

Juan Antonio Juanes Méndez, $M^{a}$ Auxiliadora Velasco Marcos, Francisco Javier Cabrero Fraile, José Miguel Sánchez Llorente y $M^{a}$ José Rodríguez Conde
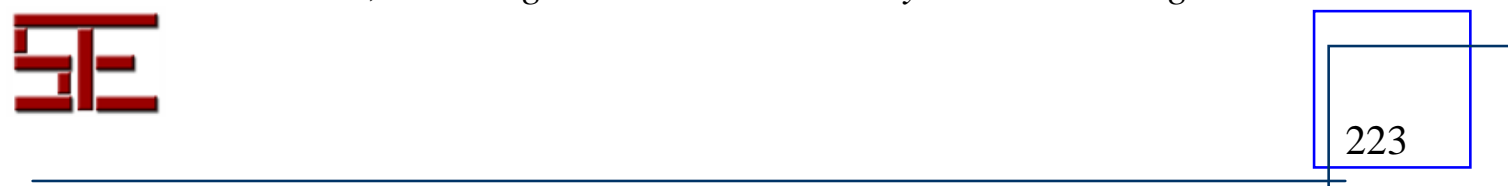


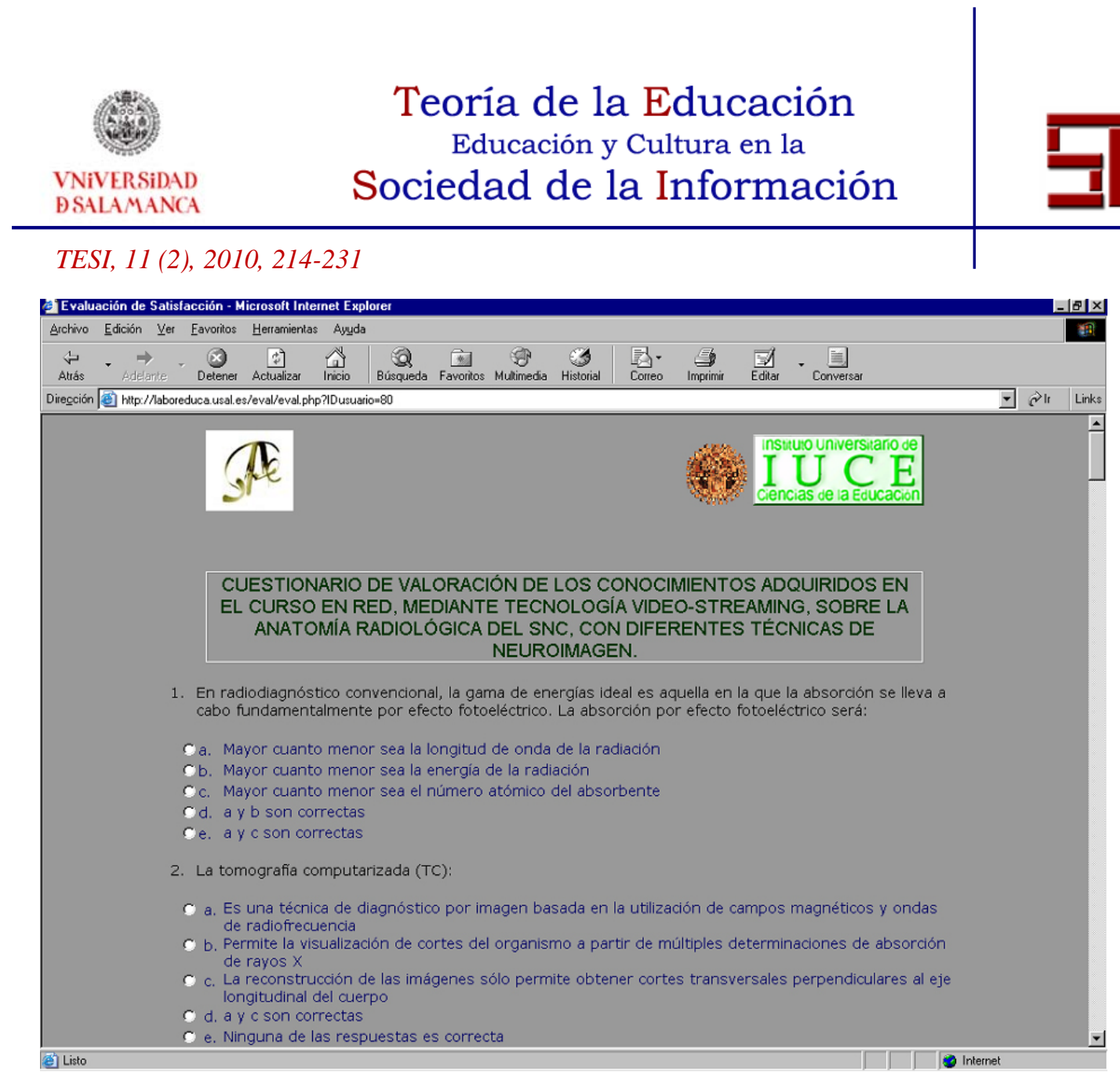

Formato tipo Test para la evaluación del contenido temático del curso, a través de red., donde el usuario recibía el número de respuestas correctas y si había superado con éxito el curso.

Después de la evaluación de los contenidos del curso se practicó una encuesta de satisfacción del mismo, de forma voluntaria; siendo el número de personas que respondieron de 45 , lo que supuso más del $80 \%$ de los matriculados en el curso. Por lo tanto el índice de respuesta lo consideramos muy satisfactorio.

El planteamiento de las preguntas formuladas a los participantes hacía alusión a que al ser una de las primeras experiencias formativas, en formato video-streaming, necesitaríamos conocer su opinión sobre el mismo. El análisis de sus opiniones nos servirá para tener un feedback sobre qué les ha parecido el curso y poder mejorar futuras ediciones del mismo o similares.

Juan Antonio Juanes Méndez, $M^{a}$ Auxiliadora Velasco Marcos, Francisco Javier Cabrero Fraile, José Miguel Sánchez Llorente v $M^{a}$ José Rodríauez Conde. 


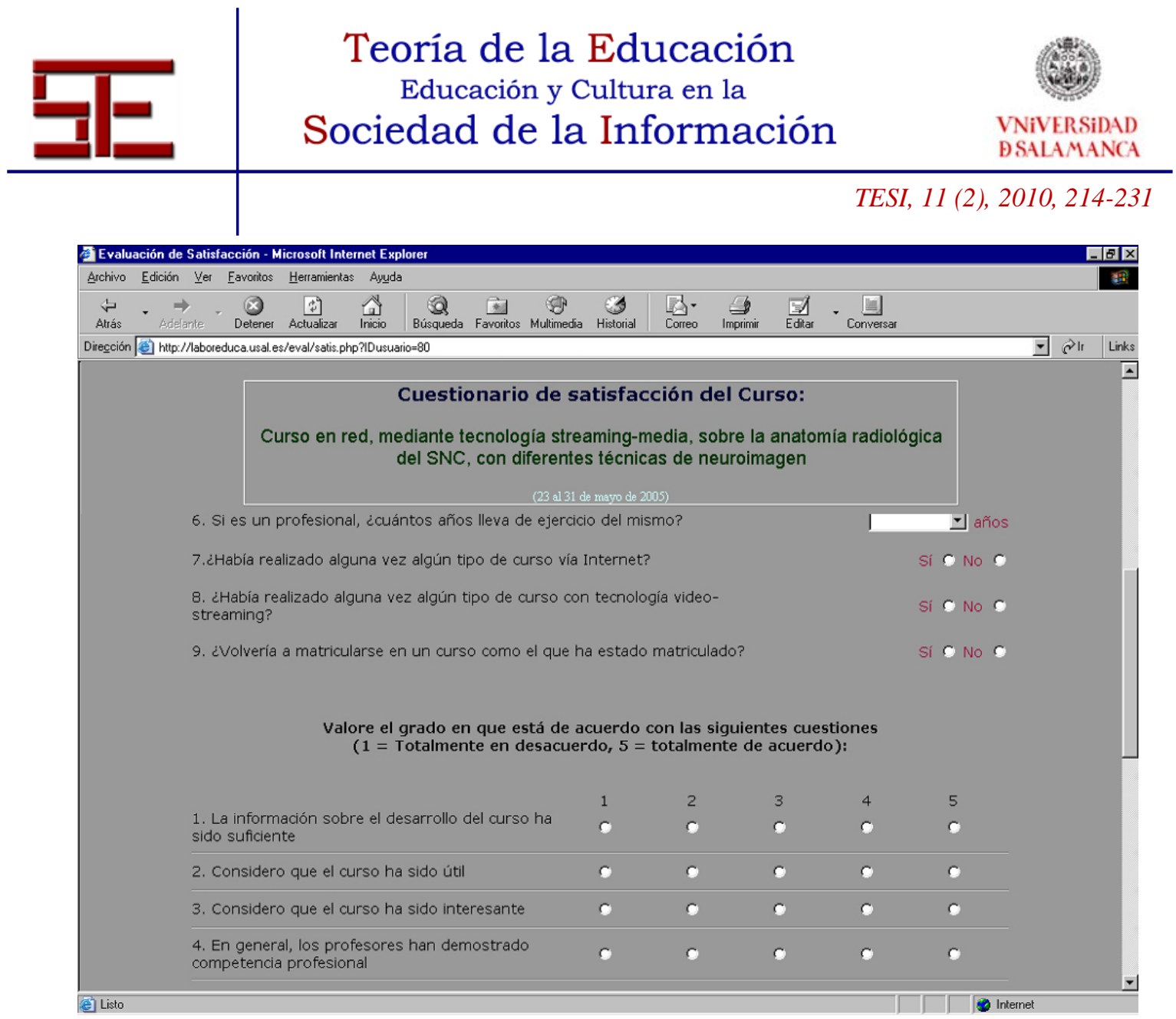

Pantalla que nos ilustra el formato de encuesta de satisfacción del curso llevada a cabo por los usuarios.

Por sexos no hubo a penas diferencias entre las personas que realizaron el curso, existiendo un $53,3 \%$ de varones y un $46,7 \%$ de mujeres.

Por otro lado, de todos los encuestados, el 37,8\% fueron estudiantes y el $46,7 \%$ personas que trabajaban; existiendo un $15,6 \%$ que estudia y trabaja a la vez.

El lugar de conexión para el desarrollo del curso fue variable: el 44,4\% fue en su casa particular; el $40 \%$ en el trabajo (Hospital, Facultad), y el 15,6\% en otros lugares (cibercafes, etc..).

En cuanto al horario de entrada al curso fue muy variable, pues se podía acceder durante las 24 horas del día, y durante los 10 días que duró todo el curso. Destaca el horario de mañana entre las 8:00 y las 15:00 horas

Juan Antonio Juanes Méndez, $M^{a}$ Auxiliadora Velasco Marcos, Francisco Javier Cabrero Fraile, José Miguel Sánchez Llorente y $M^{a}$ José Rodríguez Conde
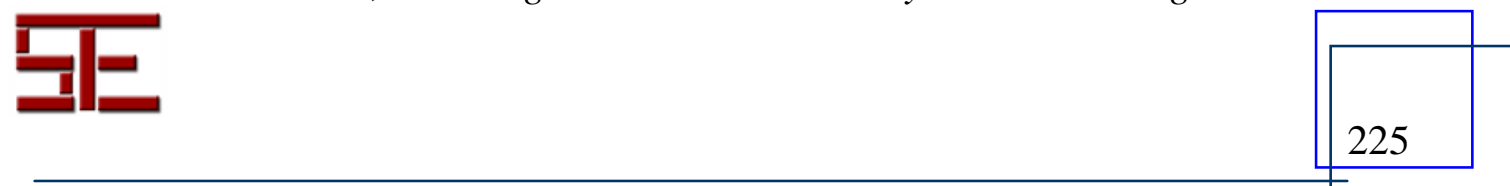


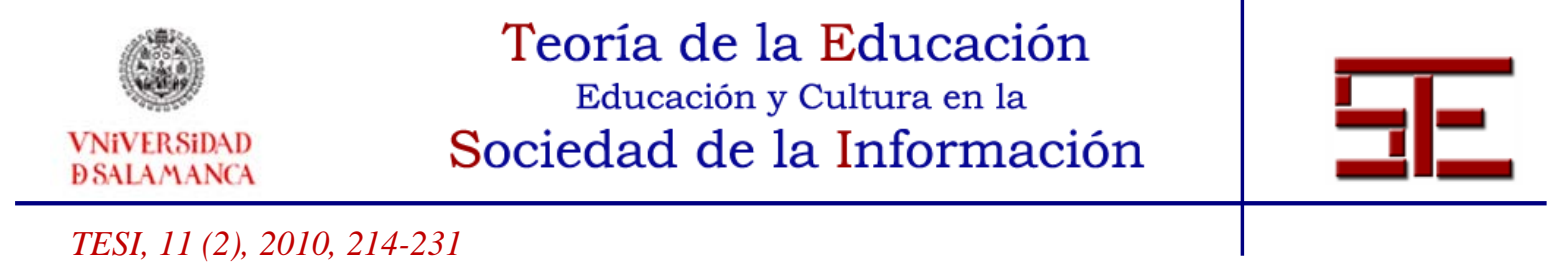

Llama la atención el dato sobre si alguna vez ha realizado un curso con tecnología video streaming, siendo el porcentaje del 93,3\% las personas que nunca han desarrollado un curso de estas características.

El dato más destacable hace referencia a la pregunta sobre si volvería a realizar un curso de estas características utilizando esta tecnología. La respuesta fue unánime, el 100\% señaló que si.

Las preguntas tenían una valoración entre 1 y 5, (siendo 1 en desacuerdo y 5 totalmente de acuerdo).

\begin{tabular}{|c|c|c|c|c|c|c|c|c|}
\hline \multirow{2}{*}{ Satisfacción hacia el curso } & \multirow{2}{*}{ Media } & \multirow{2}{*}{ Sx } & 1 & 2 & 3 & 4 & 5 & \multirow{2}{*}{$\mathbf{N}$} \\
\hline & & & $\%$ & $\%$ & $\%$ & $\%$ & $\%$ & \\
\hline $\begin{array}{l}\text { 1. La información sobre el desarrollo del } \\
\text { curso ha sido suficiente }\end{array}$ & 4,13 & ,815 & & 4,4 & 13,3 & 46,7 & 35,6 & 45 \\
\hline 2. Considero que el curso ha sido útil & 4,07 & ,780 & & 2,2 & 20,0 & 46,7 & 31,1 & 45 \\
\hline $\begin{array}{l}\text { 3. Considero que el curso ha sido } \\
\text { interesante }\end{array}$ & 4,13 & ,786 & & 6,7 & 4,4 & 57,8 & 31,1 & 45 \\
\hline $\begin{array}{l}\text { 4. En general, los profesores han } \\
\text { demostrado competencia profesional }\end{array}$ & 4,31 & ,668 & & & 11,1 & 46,7 & 42,2 & 45 \\
\hline $\begin{array}{l}\text { 5. Se han hecho aportaciones y enfoques } \\
\text { novedosos }\end{array}$ & 4,02 & ,783 & & 4,4 & 15,6 & 53,3 & 26,7 & 45 \\
\hline 6. El contenido expuesto ha sido intenso & 3,89 & ,745 & & 4,4 & 20,0 & 57,8 & 17,8 & 45 \\
\hline $\begin{array}{l}\text { 7. He aprovechado más este curso que otros } \\
\text { en los que he estado de manera } \\
\text { presencial }\end{array}$ & 3,13 & 1,392 & & 19,5 & 36,6 & 24,4 & 17,8 & 41 \\
\hline $\begin{array}{l}\text { 8. En general, estoy satisfecho con el curso } \\
\text { tal y como ha sido desarrollado }\end{array}$ & 3,87 & 1,036 & & 6,8 & 18,2 & 47,7 & 27,3 & 44 \\
\hline $\begin{array}{l}\text { 9. Recomendaría este tipo de curso (video- } \\
\text { streaming) a mis compañeros o colegas }\end{array}$ & 4,40 & ,751 & & & 15,6 & 28,9 & 55,6 & 45 \\
\hline
\end{tabular}

\section{3.- FUTURO DE LA COMUNICACIÓN Y FORMACIÓN A DISTANCIA: LA TELEINMERSIÓN.}

Un sistema de teleinmersión permite a personas situadas en distintos lugares compartir el mismo entorno virtual; es decir, en una reunión de trabajo o consulta médica, podrían interactuar, prácticamente de la misma forma a como lo harían si estuvieran esas personas en una misma habitación (Lanier, 2001).

A diferencia de las teleconferencias, que sólo sitúan a los interlocutores en pantallas de televisión, la teleinmersión da un paso más allá, pasando de las dos a las tres dimensiones. En los sistemas de videoconferencia actuales es imposible, por ejemplo, mirarse a los ojos porque la cámara y la pantalla no pueden ocupar la misma posición.

Juan Antonio Juanes Méndez, $M^{a}$ Auxiliadora Velasco Marcos, Francisco Javier Cabrero Fraile, José Miguel Sánchez Llorente v $M^{a}$ José Rodríauez Conde. 


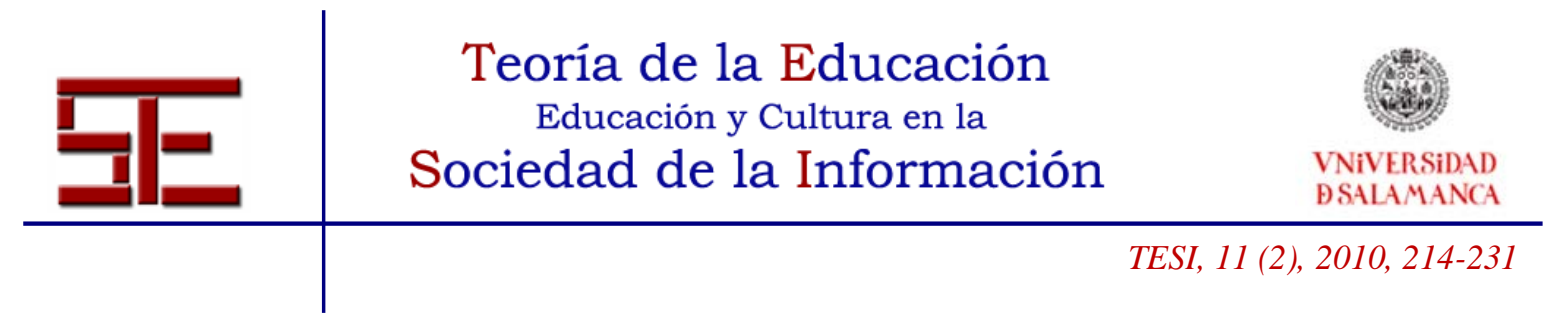

Esto suele dar un tono frío a las relaciones. Los participantes tampoco pueden adquirir consciencia de sus posiciones relativas y, por tanto, no disponen de un modo claro de llamar la atención y expresar aprobación o desacuerdo. Sin embargo, la teleinmersión constituye un nuevo sistema de interacción humana a través de técnicas digitales que confiere al usuario la ilusión de compartir un mismo espacio físico con otras personas, aunque se hallen alejadas miles de kilómetros. Para ello se combinan los procesos de visualización e interacción propios de la realidad virtual con nuevas técnicas de visión que trascienden las tradicionales limitaciones de una cámara.

Desde el punto de vista técnico, este procedimiento de comunicación a distancia, requiere un banco de cámaras digitales convencionales que capturan a los participantes en la sesión desde una gran variedad de ángulos. También se emplean telémetros láser para recoger las coordenadas de espacio. El telémetro láser es capaz de realizar medidas de distancia de forma automática como el ultrasónico, pero con mayor exactitud que el telémetro óptico. Esto se debe a que el láser se refleja en una zona muy reducida del blanco y lo va modulado. Se apunta a un objeto hasta que la imagen procedente de los objetivos se superpone en una sola. El telémetro calcula la distancia al objeto a partir de la longitud de la base y de los ángulos subtendidos entre el eje de los objetivos y la línea de la base. Cuanto mayor es la línea de la base, más preciso es el telémetro. Posteriormente un ordenador convierte las imágenes en información digital geográfica en tres dimensiones. En definitiva, la teleinmersión es un procedimiento de comunicación a distancia que une informática, video en tres dimensiones, robótica y realidad virtual, y que permite establecer una comunicación con personas distantes como si estuvieran compartiendo el mismo espacio físico. Este sistema tecnológico de comunicación a distancia requiere avances en la infraestructura de Internet, debido a las características de gran ancho de banda, bajo retardo y comunicaciones sincrónicas, dependientes del tiempo. Una vez superadas las dificultades del gran ancho de banda que requiere, esta tecnología permitirá la integración de imágenes reales, pudiendo llevar a cabo la simulación de la presencia compartida, lo que facilitará el trabajo colaborativo entre diferentes centros. Por el momento esta tecnología está sujeta a un motor más desarrollado de la actual red. Esta gran capacidad de transferencia de datos por la red se conseguirá con el desarrollo de Internet2, sobre la que ya muchas universidades americanas están trabajando. Internet2 (I2) o UCAID (University Corporation for Advanced Internet Development) es un consorcio, sin fines de lucro, que desarrolla aplicaciones y tecnologías de redes avanzadas, la mayoría para transferir información a alta velocidad. Es una red telemática desarrollada principalmente por las universidades estadounidenses, construida en fibra óptica y permite altas velocidades con una gran fiabilidad. Esta red ha sido llevada a cabo por 212 universidades de

Juan Antonio Juanes Méndez, $M^{a}$ Auxiliadora Velasco Marcos, Francisco Javier Cabrero Fraile, José Miguel Sánchez Llorente y $M^{a}$ José Rodríguez Conde

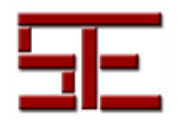




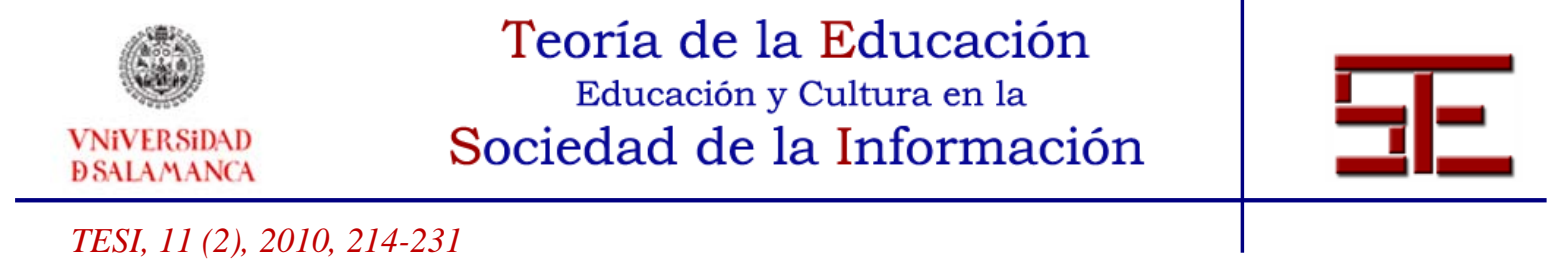

Estados Unidos y unas 60 compañías tecnológicas como Comcast, Microsoft, Intel, AMD, Sun Microsystems y Cisco Systems. Algunas de las tecnologías que han desarrollado han sido IPv6, IP Multicast y Calidad de Servicio (QoS).

Esta tecnología supone un reto apasionante para el futuro de las comunicaciones entre profesionales para el trabajo colaborativo, al crear espacios virtuales de intercambio de información, pero con la sensación de estar inmerso en un entorno cercano a realidad. Esta tecnología no se limita sólo a charlas o conferencias personales, sino que, en un futuro no muy lejano, podrá tener múltiples aplicaciones; así por ejemplo, un cirujano podrá estar presente en una sala de operaciones para aconsejar a sus colegas; o los estudiantes podrán participar de clases a distancia en cualquier universidad del mundo, etc... De momento, los prototipos llevados a cabo son muy básicos y elementales, ya que las posibilidades actuales de la red Internet son todavía muy limitadas. Representamos en las imágenes unos ejemplos gráficos de visualización, bajo tecnología teleinmersiva, establecida entre participantes de un Centro de salud y una sala del servicio de radiología del Hospital Universitario.

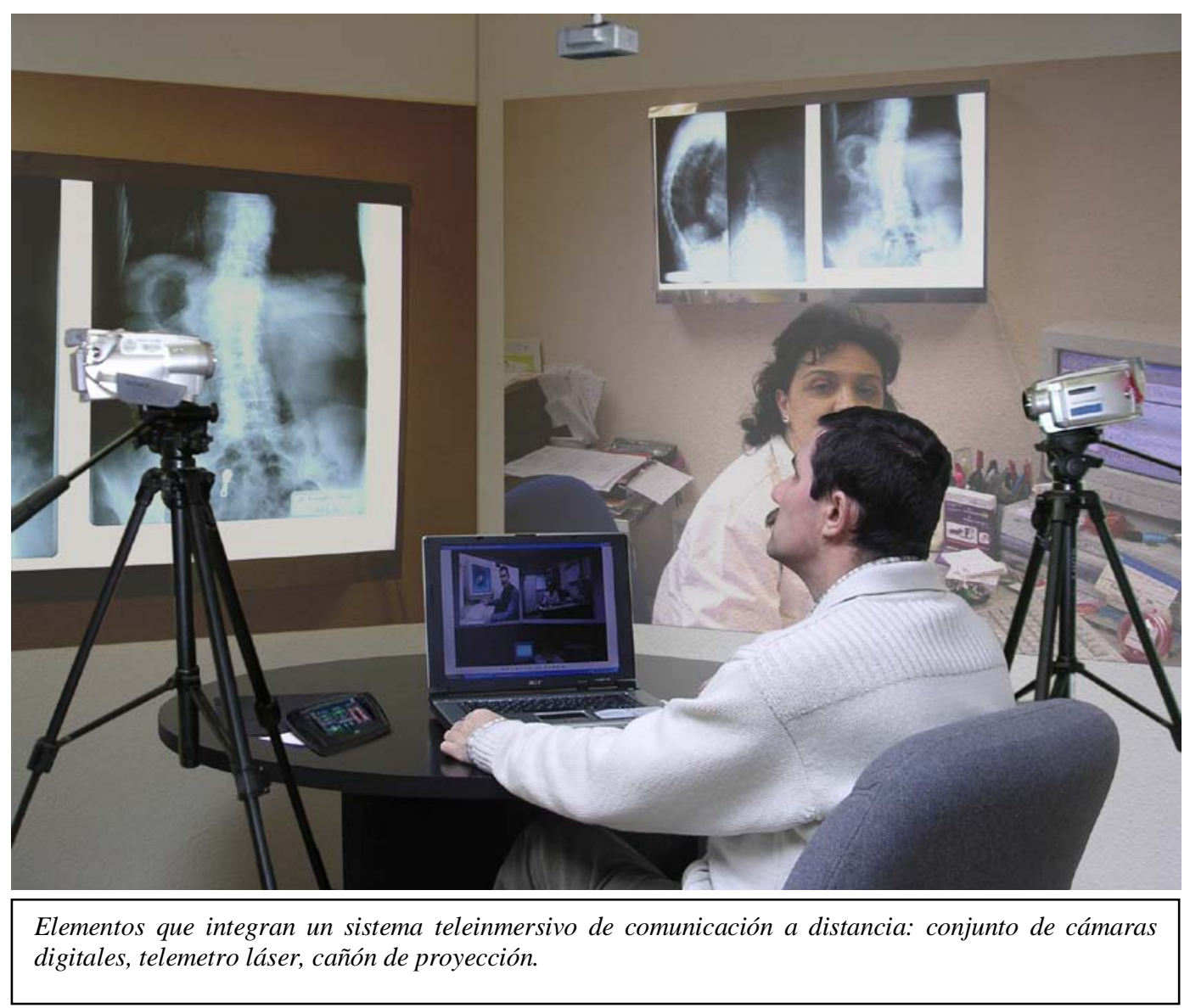

Juan Antonio Juanes Méndez, $M^{a}$ Auxiliadora Velasco

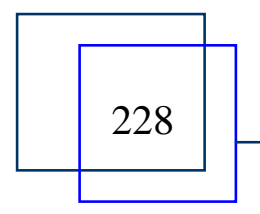

Marcos, Francisco Javier Cabrero Fraile, José Miguel Sánchez Llorente v $M^{a}$ José Rodríauez Conde. 

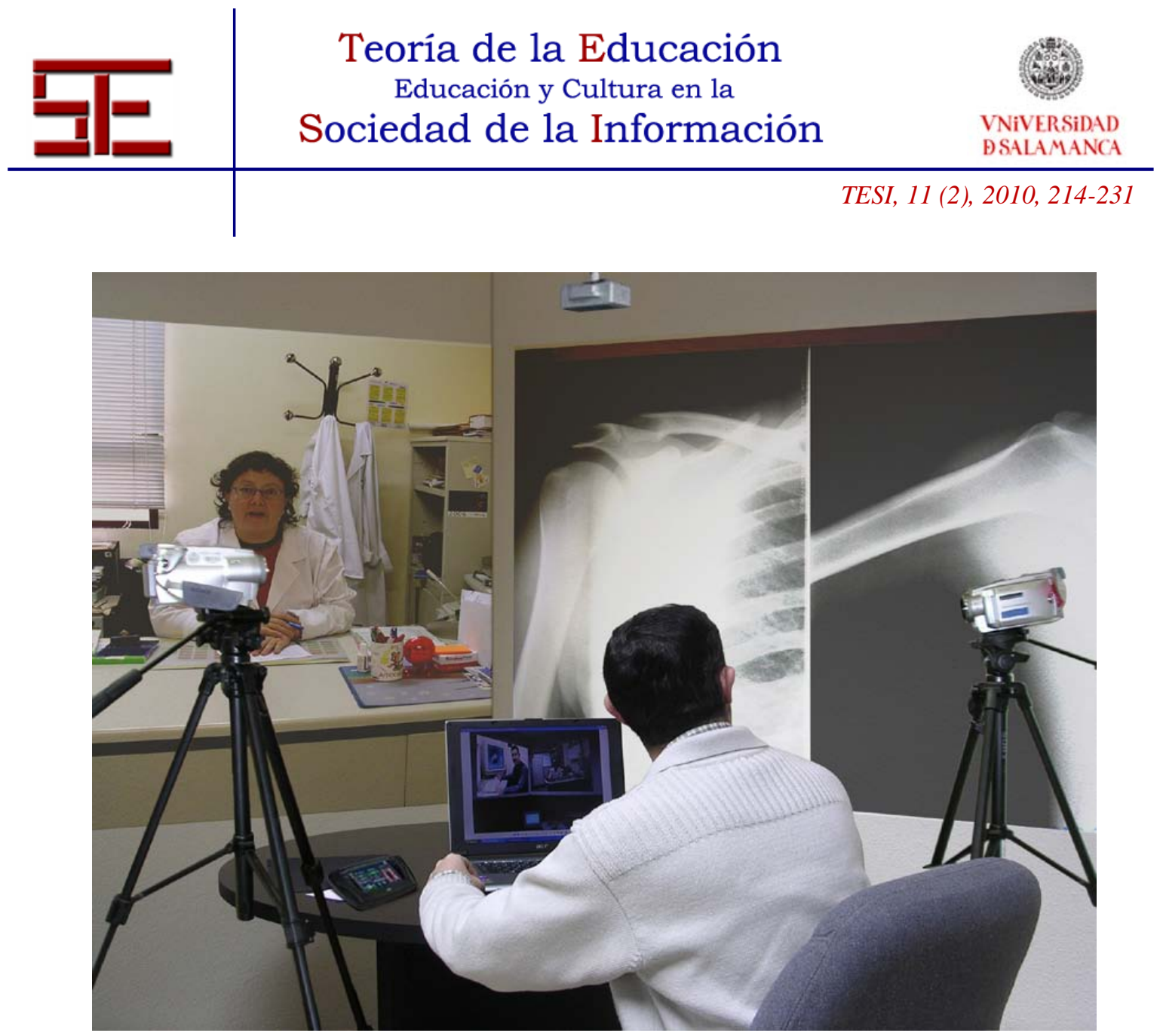

La teleinmersión constituye un sistema de interacción humana, a través de técnicas digitales de visualización, que confiere al usuario la ilusión de compartir un mismo espacio físico con otras personas, que se hallen alejadas; permitiéndoles interactuar como si estuviesen en la misma habitación. Un semicírculo de videocámaras captan la persona y su entorno desde diversos ángulos de visión.

\section{4.- CONSIDERACIONES FINALES}

El fututo de estas tecnologías es alentador. Los analistas de la industria pronostican una convergencia de la televisión y PC en la próxima década. Un "mundo broadband" marcado por el cable, ADSL, y redes satelitales junto con software/hardware avanzado, y tecnologías de soporte, proveen el ambiente necesario para la entrega de alta calidad en video a través de Internet. L atecnología video-streaming está ya revolucionando las comunicaciones entre los profesionales médicos, así como entre profesores y alumnos en cualquier disciplina universitaria; teniendo la posibilidad de comunicarnos sin importarnos las barreras de tiempo, ni las distancias. Es de resaltar que los cambios producidos, en el proceso de enseñanza-aprendizaje, dentro del marco del Espacio

Juan Antonio Juanes Méndez, $M^{a}$ Auxiliadora Velasco Marcos, Francisco Javier

Cabrero Fraile, José Miguel Sánchez Llorente y $M^{a}$ José Rodríguez Conde
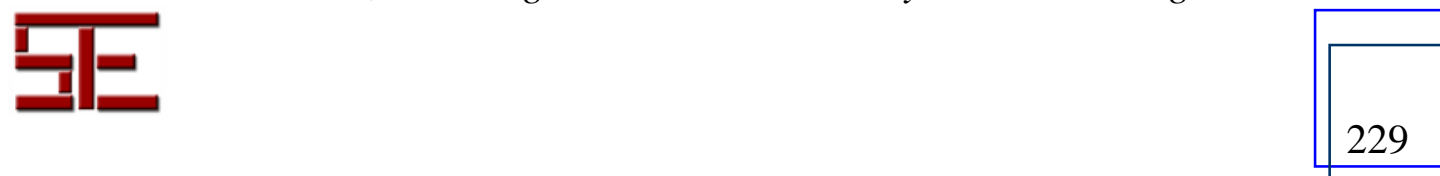


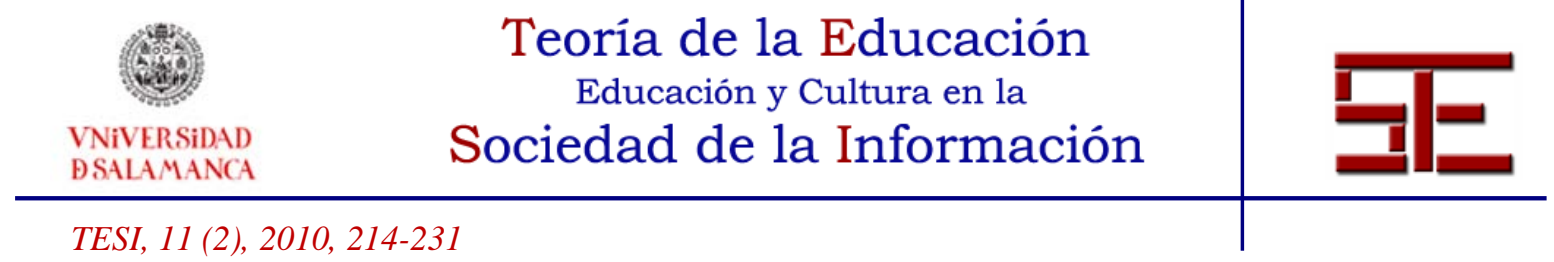

Europeo de Educación Superior (EEES), nos ha obligado a revisar la metodología de enseñanza y el diseño curricular, buscando estrategias que motiven al alumno al aprendizaje autónomo, empleando para ello herramientas didácticas, dirigidas y coordinadas por el profesor. Nos encontramos inmersos en la sociedad del conocimiento, en donde la formación a distancia se ha consolidado como un modelo virtual de aprendizaje, promovido por el uso de los medios informáticos y de las telecomunicaciones a través de Internet.

Los entornos de docencia virtuales constituyen una forma nueva de tecnología formativa, donde se deberá aprovechar todo este potencial tecnológico, para la formación de nuestros alumnos. En este sentido, la teleinmersión, en los próximos años, tendrá el potencial de cambiar significativamente los paradigmas de formación y asistencia médica a distancia. Aunque no debemos desestimar la capacidad futura de la formación a distancia, una docencia de calidad consistirá en combinar lo presencial con tecnologías complementarias de aprendizaje virtual. Pensamos que "on-line” y "face to face” deberán ir de la mano.

Este tipo de formación bimodal, aprendizaje mezclado o "blended-learning" constituye una modalidad mixta de enseñanza, que permite adaptar las formas tradicionales de docencia presencial con las modernas tecnologías de la información y comunicación.

No cabe duda de que estas tecnologías facilitarán enormemente tareas encaminadas hacia la formación y educación en salud pública y comunitaria; así como el desarrollo de programas de prevención y formación sanitaria, fácilmente transmisibles por este mecanismo de intercomunicación.

El avance tecnológico es exponencial, por lo que deberemos estar a la altura de las posibilidades que disponemos a nuestro alcance, como recursos adicionales en la docencia universitaria, y en la formación e información a distancia de pacientes. Para ello, es necesario que para la implantación de estos recursos tecnológicos exista un compromiso de las administraciones públicas a cualquier nivel, estatal, autonómico o local, para incorporar estas herramientas al terreno docente, tal y como lo vienen haciendo los países más desarrollados tecnológicamente. Sin lugar a dudas, estos sistemas cambiarán la forma de trabajar y de comunicarnos entre profesores y estudiantes universitarios y entre profesionales médicos y sus pacientes, abriendo además, nuevas perspectivas de docencia y formación médica a distancia.

Juan Antonio Juanes Méndez, $M^{a}$ Auxiliadora Velasco Marcos, Francisco Javier Cabrero Fraile, José Miguel Sánchez Llorente v $M^{a}$ José Rodríauez Conde. 


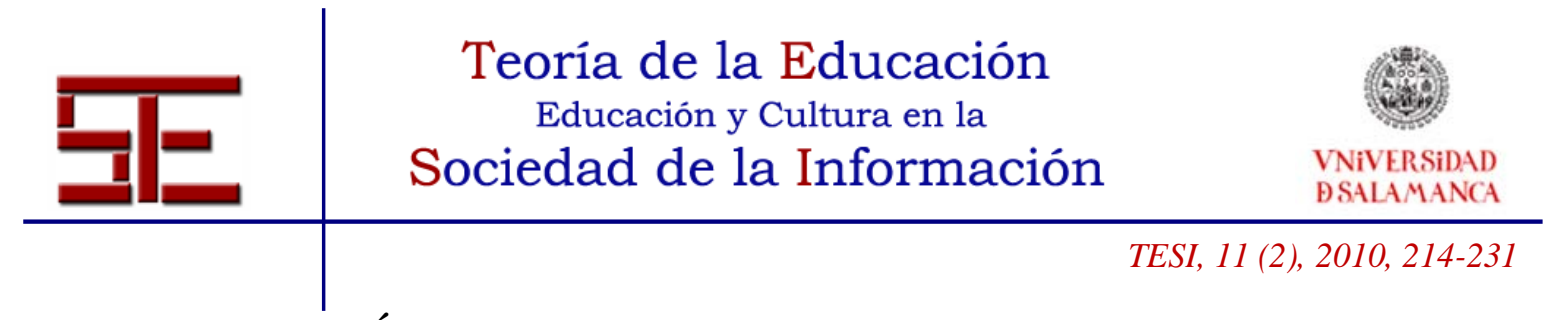

\section{5. - BIBLIOGRAFÍA.}

Ali, C., Begen, Yucel Altunbasak, Ozlem, E. Y Mehmet A, Real-Time Multiple Description and Layered Encoded Video Streaming with Optimal Diverse Routing, Georgia Institute of Technology, University of British Columbia.

Apostolopoulus, J G., Tan W. Y Wee, S. J (2002). Video Streaming: Concepts, Algorithms and Systems, Mobile and Media Systems Laboratory, HP Laboratories, Palo Alto HPL.

Badía, F. (2002). Internet: situación actual y perspectivas. Barcelona. La Caixa.

Cabaro, J. (2001). Tecnología educativa. Diseño y producción de medios. Barcelona. Paidós.

Duggy, J. (2001). El tutor on-line. La enseñanza a través de Internet. Bilbao. Deusto.

Gregory, J., Cconklin, G. S., Greenbaum, K. O., Lillevold, A. F., Lippman Y Yuryu A. R. (2001). Video Coding for Streaming Media Delivery on the Internet, 1051-8215 IEEE

Gunawardena C., Carvajal K. Y Lowe C. (2001). Critical Analysis of models and methods used to evaluate on-line learning networks. American Educational Reserach Association. Seattle, WA.

Hunter, J., Witana, V. Y Mark, A. M. (1997). A review of Video Streaming over the Internet, DST Technical Report TR97-10.

Jones, K. N., Kreisle, R., Geiss, R.W., Holliman, J. H., Lil, P.H. Y Anderson, P.G. (2002). Group for research in pathology education online resources to facilitate pathology instruction. Arch Pathol Lab Med; 126: 346-350.

Lanier, J. (2001). La teleinmersión. Investigación y Ciencia. 297: 66-75.

Martínez, F. Y Solano, I. (2003). El proceso comunicativo en situaciones virtuales. En Martínez F (comp): Redes de comunicación en la enseñanza. Barcelona. Paidós. Reid, W. A., Harvey, J., Watson, G.R., Luqmani, R., Harkin, P.J. Y Arends, M. J. (2000). Medical student appraisal of interactive computer-assisted learning programs embedded in a general pathology course. J Pathol; 191: 462-465. Szymas, J. (2000). Teleeducation and telepathology for open and distance education. Anal Cell Pathol; 21: 183-191.

Wang, Y., Ostermann, J, \& Zhang, Y. Q. (2002). Video Processing and Communications, Prentice Hall,

Juan Antonio Juanes Méndez, $M^{a}$ Auxiliadora Velasco Marcos, Francisco Javier

Cabrero Fraile, José Miguel Sánchez Llorente y $M^{a}$ José Rodríguez Conde
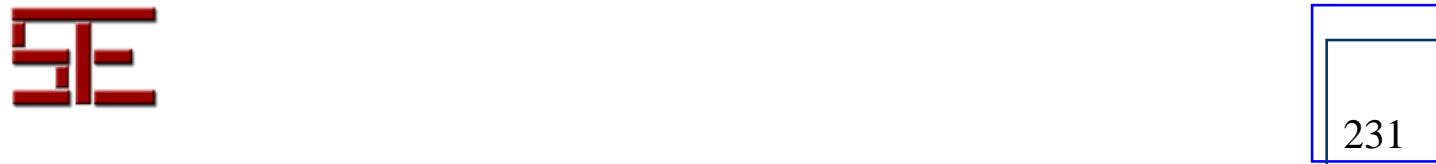


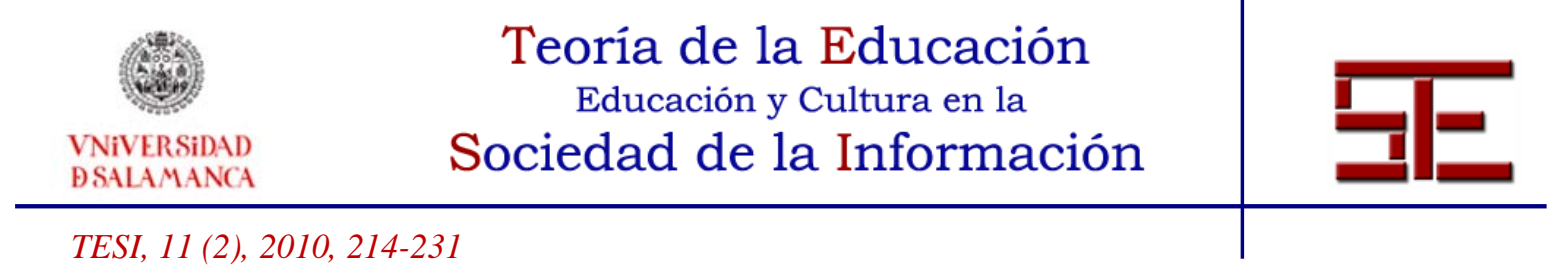

Para citar el presente artículo puede utilizar la siguiente referencia:

Juanes Méndez, J.A., Velasco Marcos, M.A., Cabrero Fraile, F.J., Sánchez Llorente J.M y Rodríguez Conde, M.J. (2010): Recursos tecnológicos audiovisuales de formación en red: sistemas streaming media y teleinmersivos, en Juanes Méndez, J. A. (Coord.) Avances tecnológicos digitales en metodologías de innovación docente en el campo de las Ciencias de la Salud en España. Revista Teoría de la Educación: Educación y Cultura en la Sociedad de la Información. Vol. 11, $\mathrm{n}^{\circ}$ 2. Universidad de Salamanca, pp. 214-231 [Fecha de consulta: $\mathrm{dd} / \mathrm{mm} /$ aaaa $]$.

http://campus.usal.es/ revistas_trabajo/index.php/revistatesi/article/view/7078/7111

Juan Antonio Juanes Méndez, $M^{a}$ Auxiliadora Velasco Marcos, Francisco Javier Cabrero Fraile, José Miguel Sánchez Llorente v $M^{a}$ José Rodríauez Conde. 